\title{
SN 1994W: Evidence of Explosive Mass Ejection a Few Years Before Explosion
}

Nikolai N. Chugai ${ }^{1}$, Robert J. Cumming ${ }^{2}$, Sergei I. Blinnikov ${ }^{3}$, Peter Lundqvist $^{2}$, Alexei V. Filippenko ${ }^{4}$, Aaron J. Barth ${ }^{4}$, Angela Bragaglia ${ }^{5}$, Douglas C. Leonard ${ }^{6}$, Thomas Matheson ${ }^{7}$, and Jesper Sollerman ${ }^{2}$

1 Institute of Astronomy, RAS, Pyatnitskaya 48, 109017 Moscow, Russia; nchugai@inasan.rssi.ru

2 Stockholm Observatory, AlbaNova University Center, 10661 Stockholm, Sweden. robert@astro.su.se, peter@astro.su.se

${ }^{3}$ ITEP, 117218 Moscow, Russia. blinn@sai.msu.su

4 Department of Astronomy, University of California at Berkeley, Berkeley, CA 94720-3411, USA.

5 Osservatorio Astronomico di Bologna, via Ranzani 1, 40127 Bologna, Italy

6 Department of Astronomy, University of Massachusetts, 710 North Pleasant Street, Amherst, MA 01003-9305, USA.

7 Harvard-Smithsonian Center for Astrophysics, Mail Stop 20, 60 Garden Street, Cambridge, MA 02138, USA.

Summary. We present and analyze spectra of the Type IIn supernova 1994W obtained between 18 and 202 days after explosion. During the first 100 days the line profiles are composed of three major components: (i) narrow $\mathrm{P}$ Cygni lines with absorption minima at $-700 \mathrm{~km} \mathrm{~s}^{-1}$; (ii) broad emission lines with blue velocity at zero intensity $\sim 4000 \mathrm{~km} \mathrm{~s}^{-1}$; (iii) broad, smooth, extended wings most apparent in $\mathrm{H} \alpha$. These components are identified with the expanding circumstellar (CS) envelope [5], shocked cool gas in the forward postshock region, and multiple Thomson scattering in the CS envelope, respectively. The absence of broad P Cygni lines from the supernova (SN) is the result of the formation of an optically thick, cool, dense shell at the interface of the ejecta and the CS envelope. Models of the SN deceleration and Thomson scattering wings are used to recover the Thomson optical depth of the CS envelope, $\tau_{\mathrm{T}} \geq 2.5$ during first month, its density $\left(n \sim 10^{9}\right.$ $\mathrm{cm}^{-3}$ ) and radial extent, $\sim(4-5) \times 10^{15} \mathrm{~cm}$. The plateau-like SN light curve, which we reproduce by a hydrodynamical model, is powered by a combination of internal energy leakage after the explosion of an extended presupernova $\left(\sim 10^{15} \mathrm{~cm}\right)$ and subsequent luminosity from circumstellar interaction. We recover the pre-explosion kinematics of the CS envelope and find it to be close to homologous expansion with outmost velocity $\approx 1100 \mathrm{~km} \mathrm{~s}^{-1}$ and a kinematic age of $\sim 1.5 \mathrm{yr}$. The high mass $\left(\approx 0.4 M_{\odot}\right)$ and kinetic energy $\left(\approx 2 \times 10^{48} \mathrm{erg}\right)$ of the CS envelope combined with small age strongly suggest that the CS envelope was explosively ejected only a few years before the SN explosion. 


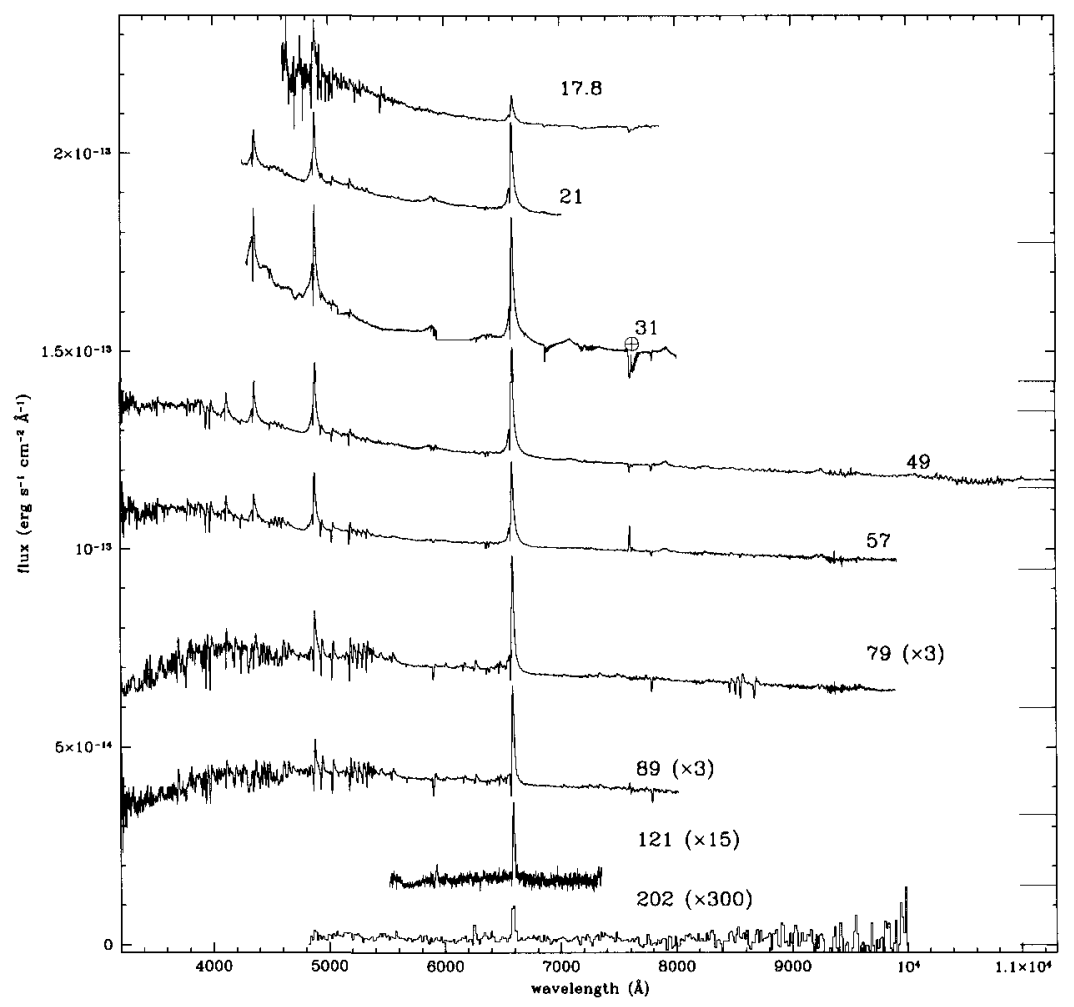

Fig. 1. Spectra of SN 1994W: days 18-202. The spectra have been shifted vertically for clarity. The ticks on the right hand side mark the zero level for each. The spectra from day 79 and later have been multiplied by a constant, noted in brackets. Note the change in relative intensity of the Balmer lines, a sign of temperature evolution.

\section{Introduction}

Type IIn supernovae are believed to arise from massive stars exploding into a dense circumstellar environment. Often highly luminous, they offer us an intriguing new window on the final stages of the progenitor's evolution.

SN 1994W, discovered on 1994 July 29 in NGC 4041, was a luminous Type IIn supernova whose light curve dropped dramatically at 110 days. The low luminosity after this point has been used to derive a very low mass of nickel in the ejecta $\left(M_{\mathrm{Ni}}<0.003 \mathrm{M}_{\odot} ;[5]\right)$. In a forthcoming paper [2], we present and analyze all the SN $1994 \mathrm{~W}$ spectra and model the circumstellar interaction.

\section{Observations}

The spectra were taken between 1994 July and 1995 February using BFOSC on the BAO 1.5-m telescope, the IDS on the Isaac Newton Telescope, ISIS 


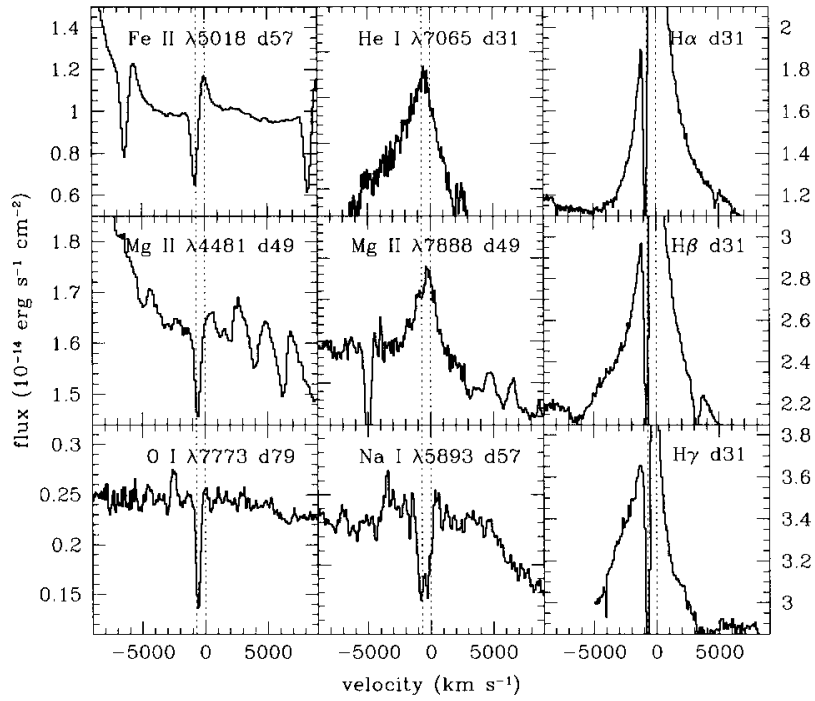

Fig. 2. Selected line profiles. The left hand panel shows examples of narrow $P$ Cygni profiles, the middle panel shows broad lines (note the extended wings in $\mathrm{Na} I$ on day 57), and the right hand panel shows the wings of the $\mathrm{H} \mathrm{I}$ lines on day 31. The dotted vertical lines mark velocities of 0 and $-700 \mathrm{~km} \mathrm{~s}^{-1}$.

on the William Herschel Telescope (La Palma), the Kast spectrograph at Lick Observatory's Shane 3-m reflector, and the LDS at the Nordic Optical Telescope on La Palma.

\section{Spectral Evolution}

No broad ejecta absorption lines are seen (Fig. 1). Together with high luminosity at maximum, this suggests an extended progenitor interacting with a circumstellar (CS) envelope, and the presence of a cool dense shell (CDS), which should form in SN II with extended envelopes [3, 4].

The spectra show persistent narrow $\mathrm{P}$ Cygni lines of $\mathrm{H}$ I with broad bases (Fig. 2). Triangular profiles with $v_{\text {FWHM }} \sim 2500 \mathrm{~km} \mathrm{~s}^{-1}$ are seen in He I and $\mathrm{Mg}$ II. Black body fits to the continuum show that the temperature declines from $15000 \mathrm{~K}$ on day 31 to $7200 \mathrm{~K}$ on day 89. From day 121 onward, only narrow emission lines are clearly seen.

We see broad lines with maximum velocity $4000 \mathrm{~km} \mathrm{~s}^{-1}$, which we take to be the expansion velocity of the CDS. We think the broad lines come from Rayleigh-Taylor fingers from the interface of the radiative forward shock and the CS envelope (Fig. 3). The persistent, narrow P Cygni lines with maximum blue velocity $1100 \mathrm{~km} \mathrm{~s}^{-1}$ indicate the maximum velocity of the circumstellar envelope, which is overrun by the ejecta at 110 days. Broad wings on $\mathrm{H}$, 


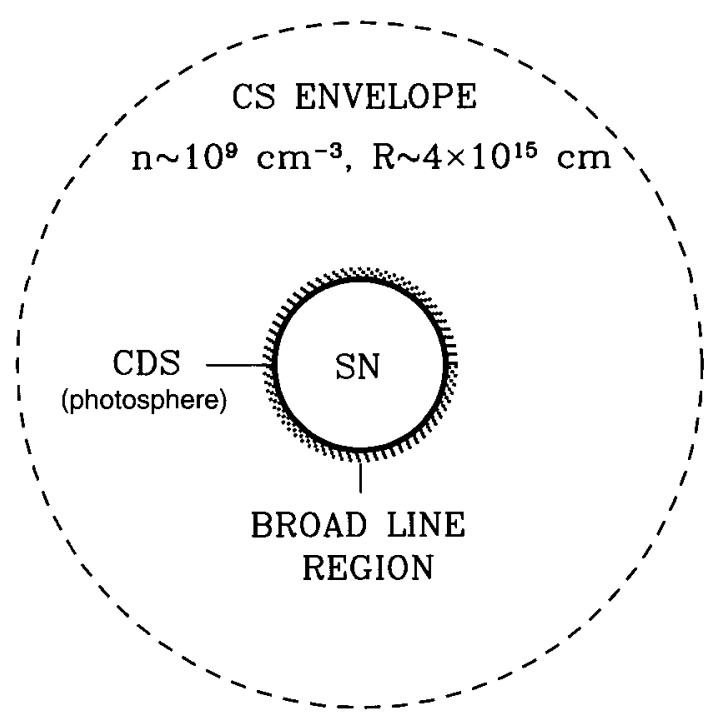

Fig. 3. A visualization of SN $1994 \mathrm{~W}$ at around day 30 . The SN ejecta is bounded by an opaque cool dense shell (CDS), which is responsible for the continuum radiation. The broad line region is a narrow mixing layer attached to the CDS, made up of Rayleigh-Taylor fragments of the CDS matter and possibly of shocked CS clouds. The SN ejecta expands into a dense CS envelope with Thomson optical depth of order unity. The CS envelope is responsible for both narrow lines and the extended Thomson wings seen in $\mathrm{H} \alpha$.

and nearly inverse Balmer decrement on day 31 point to a high optical depth for Thomson scattering, from which we estimate that the density in the CS envelope is as high as $\sim 10^{9} \mathrm{~cm}^{-3}$.

\section{Light Curve Models}

We model the broad band light curves using the multi-energy group radiation hydrodynamic code STELLA. The best fit model is for a $1.5 \times 10^{51}$ erg explosion, $M_{\mathrm{Ni}}=0.015 \mathrm{M}_{\odot}$ and $M_{\mathrm{ej}}=7 \mathrm{M}_{\odot}$ ejecta, with circumstellar envelope extending out to $R_{\text {out }}=4.5 \times 10^{15} \mathrm{~cm}$ (Fig. 4). A similar model, with the same bulk mass but no CS envelope, fails to reproduce the bright plateau up to 110 days.

\section{$5 \mathrm{H} \alpha$ Profile Model}

We have also modeled the evolution of the $\mathrm{H} \alpha$ line profile. The CS envelope is ionized by radiation from the forward shock, leading to line emission from 


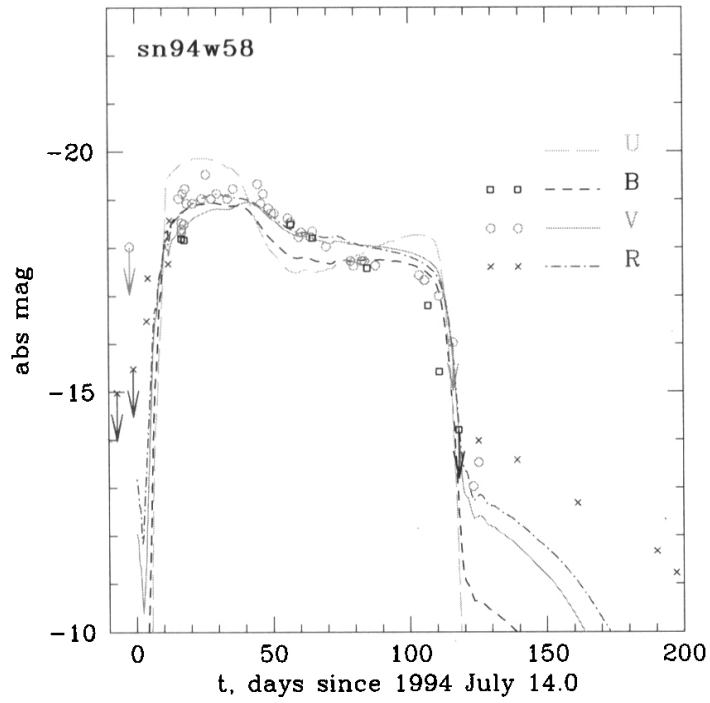

Fig. 4. Light curves for a model of SN $1994 \mathrm{~W}$ characterized by $E=1.5 \times 10^{51} \mathrm{erg}$, $M_{\mathrm{Ni}}=0.015 \mathrm{M}_{\odot}$ and $M_{\mathrm{ej}}=7 \mathrm{M}_{\odot}$.

recombination and collisional excitation. Our best fit is for a model with free expansion in the CS envelope and a boundary velocity of $1100 \mathrm{~km} \mathrm{~s}^{-1}$. This suggests a mass ejection $\sim 1.5$ yr before explosion.

Some SN IIn seem to be due to interaction with a superwind. SN 1994W, on the other hand, like SN 1995G [1], seems instead to have been preceded by a violent ejection event shortly before explosion, perhaps due to a Ne flash $[1,6]$.

\section{References}

1. N.N. Chugai, I.J. Danziger: astro-ph 0306330)

2. N.N. Chugai et al. : In preparation

3. S.W. Falk, W.D. Arnett: Astrophys. J. Suppl. 33, 515 (1977)

4. E.K. Grasberg, V.S. Imshennik, D.K. Nadyozhin: Astrophys. Space Sci. 10, 28 (1971)

5. J. Sollerman, R.J. Cumming, P. Lundqvist: Astrophys. J. 493, 933 (1998)

6. S.E. Woosley, T.A. Weaver: Ann. Rev. Astron. Astrophys. 24, 205 (1986) 